\title{
A Faster Algorithm for the Binary Epsilon Indicator Based on Orthant Minimum Search
}

\author{
Andrey Vasin \\ ITMO University \\ 49 Kronverkskiy ave. \\ Saint Petersburg, Russia \\ vasinandrey2010@gmail.com
}

\author{
Maxim Buzdalov \\ ITMO University \\ 49 Kronverkskiy ave. \\ Saint Petersburg, Russia \\ mbuzdalov@gmail.com
}

\begin{abstract}
The binary $\epsilon$-indicator is often used to assess the quality of solutions in multiobjective optimization, and to perform optimization as well. It is normally evaluated using a straightforward $\Theta(n m k)$ algorithm, where $n$ and $m$ are the number of solutions in the arguments, and $k$ is the number of objectives. This is considered to be fast compared to, for example, the hypervolume indicator, which is \#P-hard. However, there are efficient algorithms for the latter, especially for small values of $k$, while the $\epsilon$-indicator evaluation is too slow already for $n, m>10^{4}$ and for any $k$.

We present an efficient algorithm to compute the value of the binary $\epsilon$-indicator. It reduces the problem to a series of orthant minimum searches, which are solved by an appropriate algorithm. For the latter, we consider two implementations: the one based on a dynamic tree data structure, and the one based on the divide-and-conquer technique. In both cases, evaluation of the binary $\epsilon$-indicator takes $O\left((n+m) k(\log (n+m))^{\max (1, k-2)}\right)$ time. Empirical evaluation shows that the second implementation has a better performance than the first one, and both of them outperform the naive algorithm for large enough values of $n$.
\end{abstract}

\section{Keywords}

Multiobjective optimization, $\epsilon$-indicator, divide-and-conquer, orthant search, range search, performance evaluation.

\section{INTRODUCTION}

Many real-world optimization problems are multiobjective, that is, they require maximizing or minimizing several objectives, which are often conflicting. In this setup, researchers often want to know the set of Pareto-optimal solutions to the problem. However, it is often computationally or financially infeasible to obtain many Pareto-optimal solutions, so good approximations of Pareto-optimal solution sets are searched for.

In multiobjective optimization, an indicator is a function from one or more solution sets to a single number. Indicators

Permission to make digital or hard copies of part or all of this work for personal or classroom use is granted without fee provided that copies are not made or distributed for profit or commercial advantage and that copies bear this notice and the full citation on the first page. Copyrights for third-party components of this work must be honored. GECCO '16 July 20-24, 2016, Denver, CO, USA

(C) 2016 Copyright held by the owner/author(s)

ACM ISBN 978-1-4503-4206-3/16/07.

DOI: http://dx.doi.org/10.1145/2908812.2908951

\section{(c) (i)}

This work is licensed under a Creative Commons Attribution International 4.0 License. are used mainly for two purposes: for assessing the quality of a solution set, which is especially helpful in comparing outcomes of different optimizers [17], and for optimization itself $[1,2,15]$.

One of the most important indicators, and probably the best known one, is the hypervolume indicator [16]. It has many useful properties: for example, the (infinite) set of optimal solutions has the best possible value of the hypervolume indicator $[1,2]$, so this indicator is naturally suited to reduce multiobjective optimization (in the space of individual solutions) to single-objective optimization (in the space of solution sets). Many successful algorithms are based on the concept of hypervolume, including the ones for manyobjective optimization [3].

The hypervolume is \#P-hard to be computed exactly, and is NP-hard to be approximated [4]. However, there exist algorithms which are very fast for small constant dimensions (as fast as $O(n \log n)$ for two dimensions, where $n$ is the number of solutions), theoretically and practically [10,13].

However, it is argued that all unary indicators are not suited very well for performance assessment of different algorithms [17]. For this aim, several binary indicators were developed so far, including the $\epsilon$-indicator, which is the subject of the current paper. Formally, the additive binary $\epsilon$ indicator is the function on two point sets $M$ (for moving) and $F$ (for fixed) which returns the smallest value $\epsilon$, possibly a negative one, which can be added (if the objectives are to be maximized, or subtracted otherwise) to every coordinate of every point of the set $M$, so that every point in the set $F$ is weakly Pareto-dominated by at least one point $p \in M$. Similarly, a multiplicative binary $\epsilon$-indicator can be defined. Unary versions of these indicators are often defined by fixing the second argument to a certain reference point set.

The $\epsilon$-indicator possesses many useful properties, including weak dominance preservation among others. This indicator is currently used in assessing performance of various multiobjective optimizers $[11,17]$, as well as in optimization itself [15].

One of the appealing properties of the $\epsilon$-indicator is that its definition implies a very simple implementation, which consists of just three simple loops, one inside another: one for iteration over the fixed set $F$, one for iteration over the moving set $M$, and one for iteration over coordinates. This constitutes a simple $\Theta(|M| \cdot|F| \cdot k)$ algorithm with a small implementation constant (we denote as $k$ the number of objectives). This complexity is considered to be "low", especially compared to the \#P-complete hypervolume indicator. However, when the number of points grows (say, $|M|,|F| \geq 10^{4}$ ), 
this algorithm becomes slow even for $k=2$. This paper aims at improving this situation.

One of our motivation points is that with faster algorithms for $\epsilon$-indicator it will become possible to assess the quality of Pareto front approximations during the algorithm run (not only at the end). This would make it easier for the researchers to search for multiobjective algorithms with better performance at early stages of optimization.

The remainder of the paper is structured as follows. Section 2 introduces the necessary definitions. In Section 3, the problem of computation of the $\epsilon$-indicator is reduced to a certain case of the well-known orthant minimum search problem from computation geometry. Sections 4 and 5 describe efficient algorithms for the latter, which we use in this paper. Section 6 describes empirical evaluation of performance of the naive algorithm described above and of the proposed algorithm. Finally, Section 7 concludes.

\section{DEFINITIONS}

Without loss of generality, assume that we solve a multiobjective minimization problem with the number of objectives equal to $k$. In this case, Pareto dominance relation is determined on two points in the objective space as follows:

$$
\begin{aligned}
& a \prec b \leftrightarrow \forall i \in[1 ; k] a_{i} \leq b_{i} \text { and } \exists i \in[1 ; k] a_{i}<b_{i} \\
& a \preceq b \leftrightarrow \forall i \in[1 ; k] a_{i} \leq b_{i}
\end{aligned}
$$

where $a \prec b$ is called strict dominance and $a \preceq b$ is weak dominance.

The additive binary $\epsilon$-indicator, or $\epsilon$-indicator for short, is defined on two point sets $M$ (for moving) and $F$ (for fixed) and equals the smallest amount $\epsilon$ one needs to shift $M$ towards optimality such that every point from $F$ is weakly dominated by at least one point from $M$. In the case of minimization, shifting a point towards optimality by $\epsilon$ is equivalent to subtracting $\epsilon$ from each coordinate of the point. A formal definition of the $\epsilon$-indicator would have the following form:

$$
\epsilon(M, F)=\max _{f \in F} \min _{m \in M} \max _{i \in[1 ; k]}\left(m_{i}-f_{i}\right) .
$$

We finish this section with the necessary definitions regarding orthant minimum search. An orthant is simply a part of a $k$-dimensional space which consists of an intersection of $k$ half-spaces, where the $i$-th such half-space is defined by an inequality $x_{i} \geq b_{i}$ or $x_{i} \leq b_{i}$, where $b_{i}$ is a certain constant. It is a natural generalization of a ray in one dimension, quadrant in two dimensions and octant in three dimensions. In this paper, we consider only orthants directed towards positive infinity, that is, of the form $\left[b_{1} ; \infty\right) \times\left[b_{2} ; \infty\right) \times \ldots \times\left[b_{k} ; \infty\right)$.

A computation problem of orthant search considers a set of points in $k$-dimensional space, often with certain associated values. In this problem, it is needed to answer queries associated with orthants, typically directed towards either positive or negative infinity. Types of queries include finding $m$ arbitrary points belonging to the orthant, or finding the sum, the minimum or the maximum of values associated with the points belonging to the orthant. A slightly more general problem, orthogonal range search, considers not only orthants, but arbitrary ranges of the form $\left[l_{1} ; r_{1}\right] \times\left[l_{2} ; r_{2}\right] \times$ $\ldots \times\left[l_{k} ; r_{k}\right]$.

In a famous paper of Gabow, Bentley and Tarjan [8], an algorithmic framework is developed for solving several types of multidimensional geometry problems, including, among others, range searching and orthant searching for maxima and minima. It also considers problems with data structures supporting activation - a restricted type of element addition, for which it was known at the time of data structure initialization that the element is going to be added at some time. One of the data structures they proposed supports $d$-dimensional orthant searching for minima, using the activation modification operation only, and has $O\left(n(\log n)^{d-1}\right)$ preprocessing time and space, a total of $O\left(n(\log n)^{d-1} \log \log n\right)$ activation time and single query time of $O\left((\log n)^{d-1} \log \log n+\log n\right)$.

\section{REDUCTION TO ORTHANT MINIMUM SEARCH}

A naive algorithm which computes $\epsilon(M, F)$ would iterate over all points in $F$, for each such point $f$ it would check every point $m$ in $M$ and compute in $\Theta(M)$ the value of $\epsilon(m, f)$, which requires $\Theta(|M| \cdot|F| \cdot k)$ time and constant additional memory. As it is proportional to $|M| \cdot|F|$, it becomes quite slow already for $|M|,|F|>10^{4}$. In the remainder of the paper, we use $n=|M|+|F|$ where applicable, as the running times of the proposed algorithms depends mainly on the sum of these sizes.

To compute the $\epsilon$-indicator efficiently, first note that epsilon values for each point in $F$ can be computed independently of other points:

$$
\forall f \in F \text { let } v_{f}=\epsilon(M, f)=\min _{m \in M} \max _{i \in[1 ; k]}\left(m_{i}-f_{i}\right),
$$

and then the maximum of them should be taken:

$$
\epsilon(M, F)=\max _{f \in F} v_{f} .
$$

For evaluation of $\epsilon(M, f)$, we may split $M$ into arbitrary subsets $M_{1}, M_{2}, \ldots$ such that $\bigcup_{i} M_{i}=M$, solve the problem separately for each subset and take the minimum. Note that the definition of subsets can depend on $f$ arbitrarily. We define $M_{j}$ (where $1 \leq j \leq k$ ) as the set of points $m$ such that, for all $m$ in $M_{j}, \max _{i \in[1 ; k]}\left(m_{i}-f_{i}\right)=m_{j}-f_{j}$. Then, the definition of $\epsilon(M, f)$ can be rewritten as:

$$
\begin{aligned}
\epsilon(M, f) & =\min _{m \in M} \max _{i \in[1 ; k]}\left(m_{i}-f_{i}\right) \\
& =\min _{j \in[1 ; k]} \min _{m \in M_{j}} \max _{i \in[1 ; k]}\left(m_{i}-f_{i}\right) \\
& =\min _{j \in[1 ; k]} \min _{m \in M_{j}}\left(m_{j}-f_{j}\right) \\
& =\min _{j \in[1 ; k]}\left(\left(\min _{m \in M_{j}} m_{j}\right)-f_{j}\right) .
\end{aligned}
$$

The next step is to understand what the $M_{j}$ sets really are. For the chosen point $f$ and any point $m \in M_{j}$ we can write the following inequations based on the definition of $M_{j}$ :

$$
\forall i \neq j m_{j}-f_{j} \geq m_{i}-f_{i}
$$

which is equivalent to:

$$
\forall i \neq j m_{j}-m_{i} \geq f_{j}-f_{i} .
$$

Let a projection $P_{j}: \mathbf{R}^{k} \rightarrow \mathbf{R}^{k-1}$ be defined as follows:

$$
P_{j}\left(x_{1}, \ldots, x_{k}\right)=\left(x_{j}-x_{1}, \ldots, x_{j}-x_{k}\right),
$$

where the identity-zero coordinate $x_{j}-x_{j}$ is not included in the resulting point. With the use of the projection $P_{j}$, we 
1: function $\operatorname{Projection}(p, k, d)$

2: $\quad$ - builds the projection $P_{d}(p)$ given the dimension $k$

3: $\quad q \in \mathbf{R}^{k-1}$ - the projection to be constructed

4: $\quad$ for $i \in[1 ; d-1]$ do

5: $\quad q_{i} \leftarrow p_{d}-p_{i}$

6: $\quad$ for $i \in[d ; k-1]$ do

7: $\quad q_{i} \leftarrow p_{d}-p_{i+1}$

8: $\quad$ return $q$

Figure 1: The projection function which builds $P_{d}(p)$ for a point $p$ and a coordinate number $d$

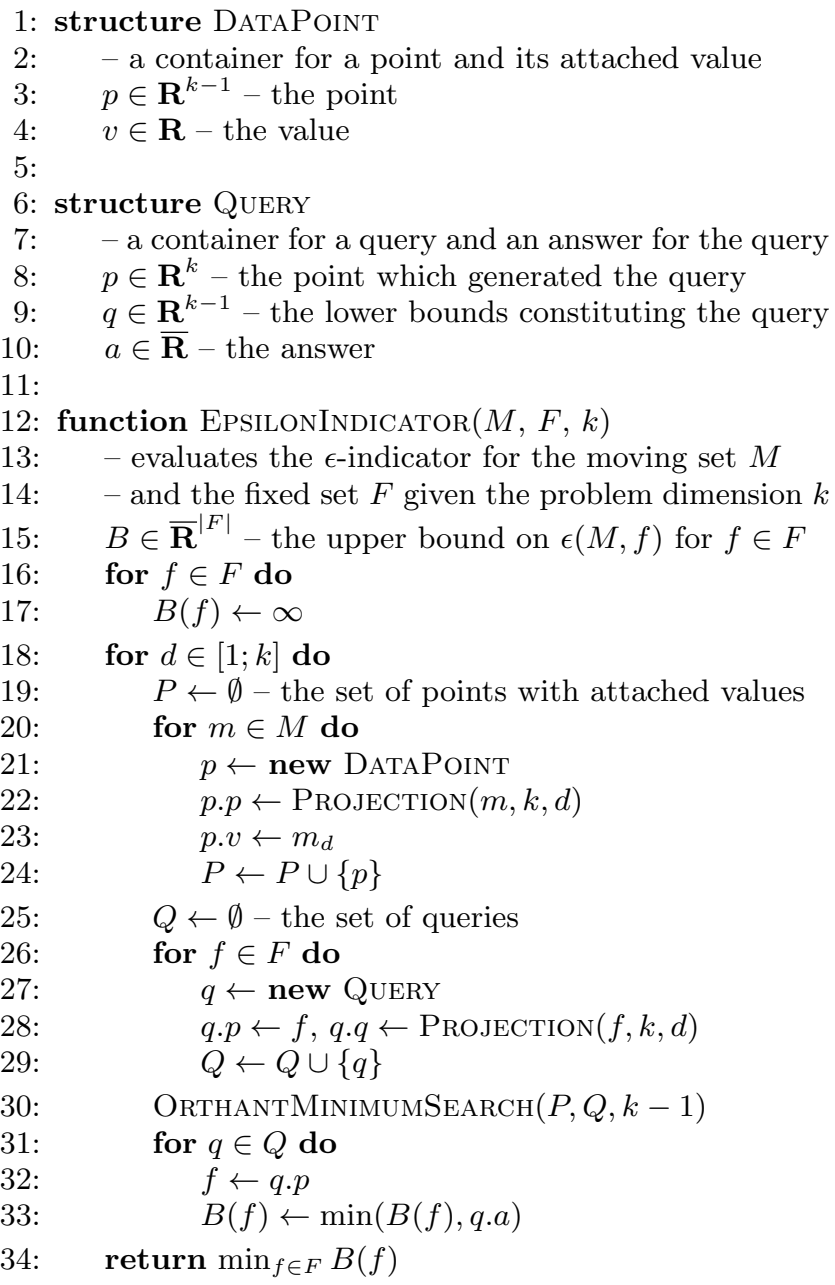

Figure 2: The algorithm of reduction of the $\epsilon-$ indicator evaluation to a series of orthant minimum searches

can rewrite (1) as follows:

$$
\forall i \neq j m_{j}-m_{i} \geq f_{j}-f_{i} \leftrightarrow P_{j}(f) \preceq P_{j}(m),
$$

so we can finally write:

$$
\epsilon(M, f)=\min _{j \in[1 ; k]}\left(\left(\min _{\substack{m \in M \\ P_{j}(f) \preceq P_{j}(m)}} m_{j}\right)-f_{j}\right) .
$$

The innermost minimum is essentially a result of an orthant minimum search in a set of points $\left\{P_{j}(m): m \in M\right\}$, where a value of $m_{j}$ is associated with a point $P_{j}(m)$. As the projection $P_{j}$ is the same for all points $f \in F$, we can build the set of projected points once for each $j$ and perform searches for all $f$. The algorithm for the projection computation is given in Fig. 1, and the overall reduction algorithm is outlined in Fig. 2.

The described algorithm can work with any algorithm for performing orthant minimum search. A key fact (which has a positive impact on the performance) is that, from the point of view of the orthant minimum search, all points and all queries are known beforehand, so any efficient offline algorithm can be used.

The total running time of the parts described in Fig. 1 and 2 is $\Theta\left(n k^{2}\right)$, and is so mainly due to building projections. The running times of the algorithms for orthant minimum search will typically dominate this running time.

In the following sections, we consider two algorithms for offline orthant minimum search which can be used inside our algorithm for the $\epsilon$-indicator evaluation. Section 4 describes an algorithm for any dimension $d$ which is based on an online (dynamic) algorithm for the dimension $d-1$. Section 5 describes an algorithm which is based on a divide-and-conquer idea very similar to the one used in efficient non-dominated sorting implementations $[5,7,9]$.

\section{DYNAMIC TREE ALGORITHM}

In [8, Theorem 3.3] it is stated that a data structure for $d$-dimensional orthant searching for maximum with activation (which can obviously be applied for minima as well) can be implemented in $O\left(n(\log n)^{d-1}\right)$ preprocessing time and space, a total of $O\left(n(\log n)^{d-1} \log \log n\right)$ activation time and single query time of $O\left((\log n)^{d-1} \log \log n+\log n\right)$. This data structure uses Van Emde Boas trees [14] to achieve $O(\log \log n)$ running times for the smallest dimensions. However, these trees either have an enormous memory overhead, or require the stored values to be compressed to small ranges of integers.

While we believe that the above mentioned problems are solvable, we chose a simpler data structure, the Fenwick tree [6]. It has the $O(\log n)$ complexity for the necessary operations, and it is very time and space efficient on modern hardware. The resulting data structure has $O\left(n(\log n)^{d}\right)$ preprocessing and activation time, $O\left(n(\log n)^{d-1}\right)$ memory requirement and $O\left((\log n)^{d}\right)$ time for a single query. Our implementation of the Fenwick tree is outlined in Fig. 3. and of the entire tree data structure in Fig. 4.

In the description of the data structure, $d=0$ is also covered for the sake of completeness, which corresponds to tracking the minimum and returning it for every query.

We use the described data structure for $(d-1)$-dimensional dynamic orthant minimum search in our algorithm for offline $d$-dimensional search to have one dimension reduced.

We do it by first sorting the data points and the query points lexicographically (first comparing the first coordinates, if equal the second ones, and so on), creating a data structure for online search on coordinates in $[2 ; d]$, and then processing both data points and query points in a common decreasing lexicographic order. As all data points are known in advance, the data structure may support activation and not insertion. Each processed data point is activated in the data structure, and for each query point the minimum is queried from the data structure. In terms of $n$ and $d$, the runtime of the preliminary sorting is $O(n d+n \log n)$ and of 
1: structure FENWICKTREE

- supports decreasing values at given keys

- and minimum queries on $[y ; \infty)$ ranges

$n \in \mathbf{N}$ - the structure size

$K \in \mathbf{R}^{n}$ - sorted known keys for binary search

$F \in \mathbf{R}^{n}$ - the Fenwick array

: function FenwickCReAte $(P)$

- creates a Fenwick tree from points $P$

$Y \leftarrow\left\{p_{2}: p \in P\right\}-$ the set of all $Y \mathrm{~s}$

$F \leftarrow$ new FenwICKTREe

$F . n \leftarrow|Y|$

$F . K \leftarrow$ UniqueSorted $(Y)$

for $i \in[1 ; F . n]$ do

$$
F . F(i) \leftarrow \infty
$$

return $F$

function FEnWICKIndex $(F, y)$

- finds an index in tree $F$ for a key $y$

$l \leftarrow 0, r \leftarrow F . n+1$

while $r-l>1$ do

$m \leftarrow\lfloor(l+r) / 2\rfloor$

if $F . K(m) \geq y$ then

$$
r \leftarrow m
$$

else

$$
l \leftarrow m
$$

return $F . n+1-r$

procedure FenwiCkUPdate $(F, y, v)$

- updates the tree $F$ at key $y$ with value $v$

$i \leftarrow \operatorname{FenwiCkIndex}(F, y)$

while $i \leq F . n$ do

$F . F(\bar{i}) \leftarrow \min (F . F(i), v)$

$i \leftarrow i+(-i$ bitwise and $i)$

function FenwickQuery $(F, y)$

- queries the tree $F$ for a minimum in $[y ; \infty)$

$i \leftarrow \operatorname{FenwickIndex}(F, y)$

$v \leftarrow \infty$

while $i>0$ do

$v \leftarrow \min (v, F . F(i))$

$i \leftarrow i-(-i$ bitwise and $i)$

return $v$

Figure 3: A Fenwick tree implementation with range compression used in this paper

the subsequent step is $O\left(n(\log n)^{d-1}\right)$. The outline of the algorithm is given in Fig. 5 .

For computing the value of the $\epsilon$-indicator for $k$-dimensional points, we perform $k$ different $(k-1)$-dimensional offline orthant minimum searches. The total running time is thus $O\left(k \cdot\left(n k+n \log n+n(\log n)^{k-2}\right)\right)$

\section{DIVIDE-AND-CONQUER ALGORITHM}

In this section, we describe an alternative algorithm for offline orthant minimum search. This algorithm is inspired by theoretically worst-case efficient algorithms for non-dominated sorting $[5,7,9]$, as non-dominated sorting itself can be seen as an application of offline orthant search.
1: structure TREE

$d \in \mathbf{Z}$ - the tree's dimension

$l \in \overline{\mathbf{R}}$ - the minimum coordinate stored

$m \in \mathbf{R}$ - the coordinate which separates children

$L, R$ : TREE - the left and right children

$B$ : TREE - the tree for $d-1$

$F:$ FENWICKTREE - the Fenwick tree for $d=1$

function TreeCreate $(P, d)$

- creates a tree from points $P$ and dimension $d$

$T \leftarrow$ new TREE

$T . d \leftarrow d, T . l \leftarrow \infty$

if $d=1$ then

$T . F \leftarrow$ FenwickCReate $(P)$

else if $d>1$ then

$T . B \leftarrow$ TreeCreate $(P, d-1)$

$T . l \leftarrow \min \left\{p_{d}: p \in P\right\}$

$T . m \leftarrow \operatorname{Median}\left(\left\{p_{d}: p \in P\right\}\right)$

$P_{L}, P_{M}, P_{R} \leftarrow \operatorname{Split}(P, T . m)$

if $P_{L} \neq \emptyset$ then

$T . L \leftarrow$ TreeCreate $\left(P_{L}, d\right)$

$P_{R} \leftarrow P_{M} \cup P_{R}$

if $P_{R} \neq \emptyset$ then

$T . R \leftarrow$ TreeCreate $\left(P_{R}, d\right)$

return $T$

function TreeActivate $(T, p, v)$

- activates a point $p$ with value $v$ in tree $T$

if $T . d=0$ then

$T . l \leftarrow \min (T . l, v)$

else if $T . d=1$ then

FENWiCKUpdate $\left(T . F, p_{2}, v\right)$

else

TreeActivate $(T . B, p)$

if $p_{T . d+1}<T . m$ then

TreeActivate $(T . L, p)$

else if $T . R \neq$ NULL and $p_{T . d+1}>T . l$ then

TreeActivate $(T . R, p)$

9:

40: function TreeQuery $(T, p)$

- perform a query for a point $p$

if $T . d=0$ then

return $T . l$

else if $T . d=1$ then

return FENWICKQUERY $\left(T . F, p_{2}\right)$ else

if $p_{T . d+1}<=T . l$ then

else

return TreeQuery $(T . B, p)$

$v \leftarrow \infty$

if $T . R \neq$ NULL then

$$
v \leftarrow \operatorname{TreeQuery}(T . R, p)
$$

if $p_{T . d+1}<T . m$ then

$v \leftarrow \min (v, \operatorname{TreEQuery}(T . L, p)$

return $v$

Figure 4: A tree data structure with activation support for dynamic orthant minimum search

To get the idea how the divide-and-conquer approach works for this problem, consider Fig. 6. In this two-dimensional fig- 
1: procedure OrthantMinimumSearch $(P, Q, d)$

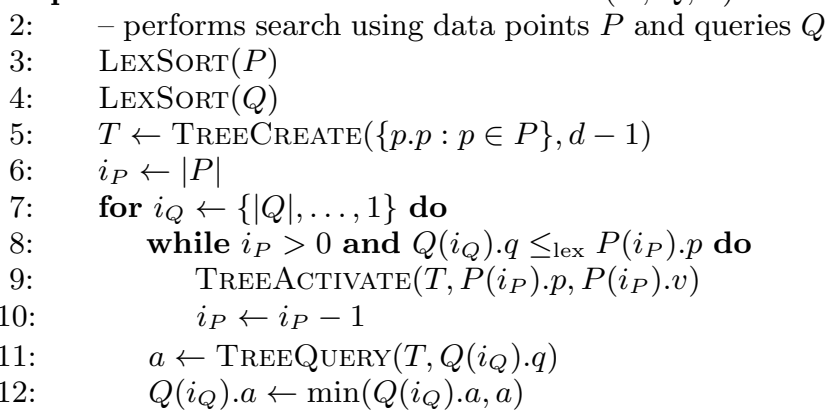

Figure 5: The main orthant minimum search procedure using the dynamic tree data structure

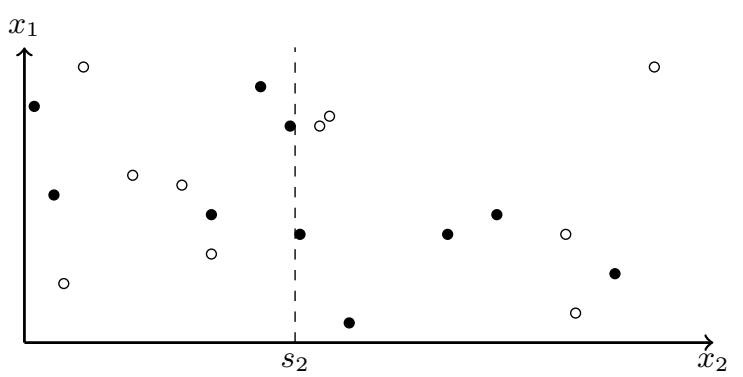

Figure 6: An illustration of the divide-and-conquer approach for orthant minimum search

ure, white points correspond to queries, while black points correspond to data points. A vertical line $x_{2}=s_{2}$ splits the set of white points $Q$ into two sets $Q_{L}$ and $Q_{R}$, and the set of black points $P$ into two sets $P_{L}$ and $P_{R}$, such that:

- $\forall q \in Q_{L} q_{2}<s_{2} ; \forall q \in Q_{R} q_{2}>s_{2}$;

- $\forall p \in P_{L} p_{2}<s_{2} ; \forall p \in P_{R} p_{2}>s_{2}$;

- $\left|Q_{L}\right|+\left|P_{L}\right|=\left|Q_{R}\right|+\left|P_{R}\right|$.

One can easily see that for every query point $q \in Q_{R}$, one can consider the points $p \in P_{R}$ only, because for every $p \in P_{L}$ it holds that $p_{2}<q_{2}$ and $p$ is not covered by the query $q$. What is more, for every query $q \in Q_{L}$ and every point $p \in P_{R}$ it holds that $q_{2}<p_{2}$, so the algorithm does not have to check this condition anymore.

If we call our procedure $\operatorname{ALgo}(P, Q, d)$, where $d$ is the current dimension, then its main branch, after finding a median (the value $s_{2}$ in the example above) and making splits, consists of three recursive calls, namely, $\operatorname{ALgO}\left(P_{L}, Q_{L}, d\right)$, $\operatorname{Algo}\left(P_{R}, Q_{R}, d\right), \operatorname{Algo}\left(P_{R}, Q_{L}, d-1\right)$. If the running time can be expressed as $T(\operatorname{Algo}(P, Q, d))=T_{d}(|P|+|Q|)$, we can estimate it as follows:

$$
T_{d}(n) \leq O(n)+2 T_{d}(n / 2)+T_{d-1}(n / 2),
$$

which, provided that $T_{d-1}(n)=O\left(n(\log n)^{d-2}\right)$, gives us $T_{d}(n)=O\left(n(\log n)^{d-1}\right)$.

The simplified description above misses three points:

1. The recursion needs to be terminated for small values of $d$. We deal with it by solving the problem for $d=2$ in $O(n \log n)$ using the idea from Section 4, namely, sorting all the points lexicographically and using the Fenwick tree (see Fig. 3) for handling one-dimensional queries.

2. Special handling is necessary for the boundary cases $|P| \leq 1$ or $|Q| \leq 1$. If one of the sets is empty, nothing should be done. The case of unit size is handled by simply testing all points with all queries in remaining coordinates $[1 ; d]$. The running time of this case would be $O(|P| \cdot|Q| \cdot d)$, which is the same asymptotically as $O(\max (|P|,|Q|) \cdot d)$ if $|P|=1$ or $|Q|=1$.

3. Treatment of equal coordinate values. There can be many values equal to the median, which may render the split uneven. This issue is solved by splitting each set $S$ not in two but in three sets: $S_{L}$ with coordinates less than the median, $S_{M}$ with coordinates equal to the median and $S_{R}$ with coordinates greater than the median. This ensures that, when we split the sets $P$ and $Q$ at their common median in the current coordinate, the subsets will satisfy inequations $2\left(\left|P_{L}\right|+\left|Q_{L}\right|\right) \leq$ $|P|+|Q|$ and $2\left(\left|P_{R}\right|+\left|Q_{R}\right|\right) \leq|P|+|Q|$, which is crucial for the running time estimation.

The overall algorithm is outlined in Fig. 7. Its running time is $O\left(n \log n+n d+n(\log n)^{d-1}\right)$ for a $d$-dimensional problem, and it requires $O(n+d)$ additional memory only.

\section{EMPIRICAL EVALUATION}

We conducted empirical evaluation of the proposed algorithm with the orthant minimum search algorithms described in Sections 4 and 5 and of the naive algorithm for computing the $\epsilon$-indicator. We used dimensions in the range $[2 ; 6]$. We also used equal sizes of both moving and fixed point sets. These sizes were taken from the set $\{100,310$, $1000,3100,10000,31000\}$ to have the ratio between the consecutive sizes be approximately equal to $\sqrt{10}$. The lower bound is chosen to be equal to one of the typical generation sizes in evolutionary algorithms, while the upper bound is chosen for the sole reason of having feasible running times of experiments.

To generate points, we used two ways: uniformly random points from the $[0 ; 1]^{k}$ hypercube, and random coplanar points residing in the $x_{1}+\ldots+x_{k}=k$ hyperplane, with coordinates $[2 ; k]$ taken uniformly at random from the $[0 ; 1]$ range. By using random points from the hypercube, we hope to approximate the behavior of all the algorithms in initial stages of optimization. Similarly, we hope to approximate the behaviour in later stages of optimization, when the points have almost converged to the Pareto optimal set, by using coplanar points. For each configuration, we report the average running time over 20 independent runs.

The plots for random points are presented in Fig. 8-12 for dimensions 2-6 correspondingly, and the raw data in Tables 1-5. All times are given in seconds, all figures have logarithmic scales in both axes. We present only the results for the random points, as the data for the coplanar points differs only by at most $10 \%$ with two exceptions $(16 \%$ for the tree-based algorithm, $n=310, k=2$ and $11 \%$ for the same algorithm, $n=100, k=5$ ). This in turn suggests that the speedup or slowdown depends only on the number of points and the dimension.

From analyzing slopes of the plots, one can immediately see that the proposed algorithm, with both algorithms used 
1: procedure AnswerQueries $(P, Q, d)$

2: $\quad$ if $P=\emptyset$ or $Q=\emptyset$ then

3: $\quad$ return

4: $\quad$ else if $|P|=1$ or $|Q|=1$ then

$5:$

$6:$

$7:$

8:

9:

10:

11:

12:

13:

14:

15:

16:

$17:$

18:

19:

20:

21:

22 :

23:

24:

25:

26:

27:

28:

29:

30:

31:

32:

33:

34:

35:

$36:$

37:

38:

39:

$$
\begin{gathered}
\text { for } q \in Q, p \in P \text { do } \\
\text { if } q . q \preceq_{d} p . q \text { then } \\
q . a \leftarrow \min (q . a, p . v)
\end{gathered}
$$

else if $d=2$ then

$F \leftarrow \operatorname{FenwiCkCreate}(\{p \cdot p: p \in P\})$

$i_{P} \leftarrow|P|$

for $i_{Q} \leftarrow\{|Q|, \ldots, 1\}$ do

while $i_{P}>0$ and $Q\left(i_{Q}\right) \cdot q \leq_{\operatorname{lex}_{2}} P\left(i_{P}\right) \cdot p$ do FenwickUpdate $\left(F, P\left(i_{P}\right) \cdot p_{2}, P\left(i_{P}\right) \cdot v\right)$ $i_{P} \leftarrow i_{P}-1$

$a \leftarrow \operatorname{FenwiCKQueRY}\left(T, Q\left(i_{Q}\right) \cdot q_{2}\right)$

$Q\left(i_{Q}\right) . a \leftarrow \min \left(Q\left(i_{Q}\right) \cdot a, a\right)$

else if $\max _{q \in Q} q . q_{d} \leq \min _{p \in P} p \cdot p_{d}$ then

else

$\operatorname{AnswerQueries}(P, Q, d-1)$

$m \leftarrow \operatorname{Median}\left(\left\{p \cdot p_{d}: p \in P\right\} \cup\left\{q . q_{d}: q \in Q\right\}\right)$

$P_{L}, P_{M}, P_{R} \leftarrow \operatorname{Split}(P, m, d)$

$Q_{L}, Q_{M}, Q_{R} \leftarrow \operatorname{Split}(Q, m, d)$

ANSWERQueries $\left(P_{R}, Q_{R}, d\right)$

AnswerQueries $\left(P_{L}, Q_{L}, d\right)$

AnswerQueries $\left(P_{M} \cup P_{R}, Q_{L} \cup Q_{M}, d-1\right)$

procedure OrthantminimumSearch $(P, Q, d)$

- performs search using data points $P$ and queries $Q$

$\operatorname{LExSORT}(P)$

$\operatorname{LExSORT}(Q)$

if $d=1$ then

$i_{P} \leftarrow|P|$

$m \leftarrow \infty$

for $i_{Q} \leftarrow\{|Q|, \ldots, 1\}$ do

while $i_{P}>0$ and $Q\left(i_{Q}\right) \cdot q_{1} \leq P\left(i_{P}\right) \cdot p_{1}$ do

$m \leftarrow \min \left(m, P\left(i_{P}\right) \cdot v\right)$

$i_{P} \leftarrow i_{P}-1$

$Q\left(i_{Q}\right) . a \leftarrow \min \left(Q\left(i_{Q}\right) \cdot a, m\right)$

else

$\operatorname{AnswerQueries}(P, Q, d)$

Figure 7: The orthant minimum search procedure using the divide-and-conquer algorithm

for orthant minimum search, indeed has a better asymptotic behavior compared to the naive algorithm.

For dimensions 2 and 3, the proposed algorithm is seen to outperform the naive algorithm for all considered problem sizes, although for $n=100$ and $k=3$ the running times are almost coinciding. For these dimensions, there is no visible difference between the orthant minimum search algorithms.

For higher dimensions, two phenomena can be seen. First, the higher the dimension, the worse the dynamic tree implementation of the proposed algorithm behaves compared to the divide-and-conquer implementation: the plots remain parallel, but the difference in vertical offsets grows. Second, the proposed algorithm (here we consider the divide-andconquer implementation) starts to outperform the naive algorithm starting from a certain problem size only: from approximately 350 for $k=4$, from 1000 for $k=5$, and from 3000 for $k=6$ (numbers are slightly interpolated).

\section{CONCLUSION}

We presented an algorithm for faster computation of the additive binary $\epsilon$-indicator. This algorithm is based on reduction of this problem to a series of orthant minimum searches. To perform the latter, we used two approaches: the one based on the classic tree-based data structure for orthant and range queries, and the one based on the multidimensional divide-and-conquer idea.

Empirical evaluation showed that the proposed algorithm works faster than the naive one, especially in small dimensions $(k=2$ or 3$)$, but in higher dimensions as well for larger $n$ due to better asymptotic of the running time. For the orthant minimum search implementations, the one based on the divide-and-conquer idea performs better, which is noticeable especially in higher dimensions. The running time ratio of the naive algorithm to the divide-and-conquer one reaches almost 15 on $n=1000$ and $k=2$, almost 6 on $n=31000, k=6$, and almost 316 (!) on $n=31000, k=2$.

The results of this paper are obviously not limited to the additive $\epsilon$-indicator: the multiplicative $\epsilon$-indicator, defined first in [17], can be computed using the same algorithm after applying logarithm to all coordinates.

This paper basically allows researchers to assess the quality of Pareto front approximations not only at the end of the algorithm run, but during the run as well, without incurring a big performance overhead. This, in turn, may make it easier to search for multiobjective optimizers with not only good overall solution quality, but with good "fixed-budget" performance as well, which has immediate application for the practitioners.

Algorithm developers may also benefit from the results of this paper. For example, algorithms similar to the IBEA algorithm [15] use queries to the binary indicator of the form $I(X \backslash\{x\},\{x\})$ to assign fitness to the solutions. In fact, IBEA itself uses a different, but computationally very similar measure:

$$
F(x)=\sum_{y \in X \backslash\{x\}}-e^{-I(\{y\},\{x\}) / \kappa} .
$$

These algorithms can be made faster than the obvious $O\left(n^{2} k\right)$ implementation (where $n=|X|$ and $k$ is the dimension) by using the ideas presented in this paper. More precisely, we can process all $F(x)$ queries for $x \in X$ simultaneously in $O\left(n k(\log n)^{k-2}\right)$ by implementing an equivalent of the NDHELPERA procedure from [5], while the ANSwERQUERIES procedure from this paper (Fig. 7) is equivalent to the NDHelPERB procedure. We intend to test this idea in the very near future.

Another important component for the future work is to adapt the ideas of this algorithm to other indicators used in multiobjective optimization, and to make these algorithms, and the similar ones, even faster. One degree of freedom, which was not exploited in this paper to the very end, is to use advanced techniques, such as Van Emde Boas trees or fractional cascading, at the low levels of the proposed algorithms and data structures. While we have some sceptical expectations about using Van Emde Boas trees in the classic way in the tree-based implementation considered in this paper, as the hidden constant grows significantly with $k$, a more appropriate application may speed up the algorithm significantly. One of the inspirations for this work would be a paper of Nekrich [12] which explains how to perform non-dominated sorting in three dimensions in $o(n \log n)$. 


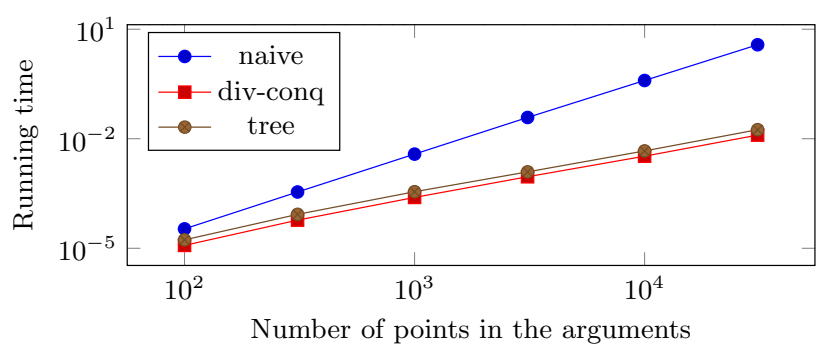

Figure 8: Plots for random points, $k=2$

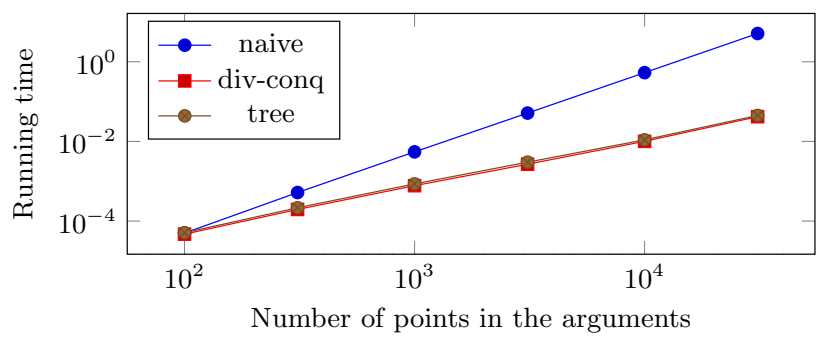

Figure 9: Plots for random points, $k=3$

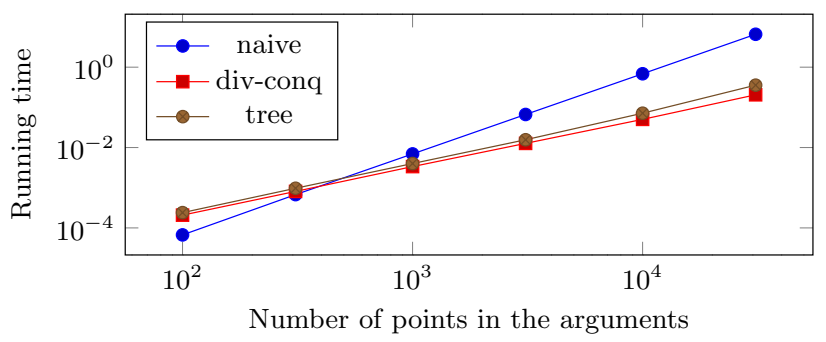

Figure 10: Plots for random points, $k=4$

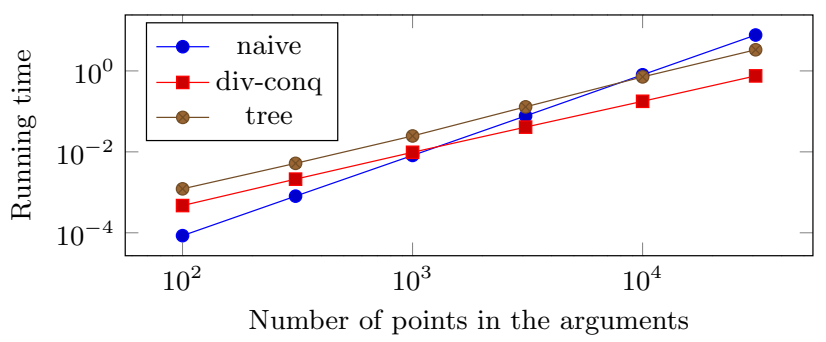

Figure 11: Plots for random points, $k=5$

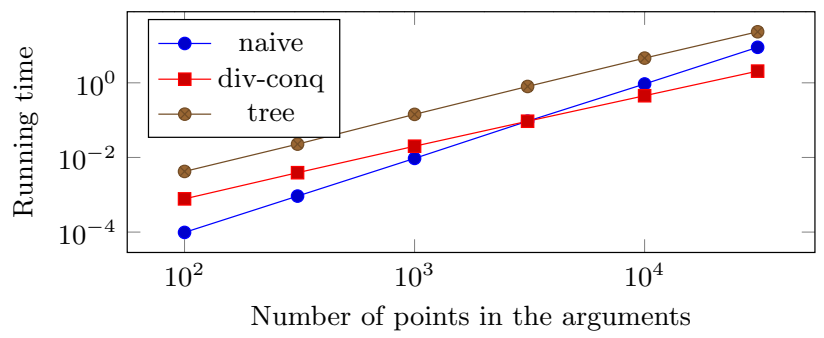

Figure 12: Plots for random points, $k=6$

\begin{tabular}{|r|c|c|c|}
\hline$n$ & naive & tree & div-conq \\
\hline 100 & $3.40 \cdot 10^{-5}$ & $1.70 \cdot 10^{-5}$ & $1.20 \cdot 10^{-5}$ \\
\hline 310 & $3.48 \cdot 10^{-4}$ & $8.50 \cdot 10^{-5}$ & $5.90 \cdot 10^{-5}$ \\
\hline 1000 & $3.80 \cdot 10^{-3}$ & $3.54 \cdot 10^{-4}$ & $2.47 \cdot 10^{-4}$ \\
\hline 3100 & $3.83 \cdot 10^{-2}$ & $1.24 \cdot 10^{-3}$ & $9.10 \cdot 10^{-4}$ \\
\hline 10000 & $3.94 \cdot 10^{-1}$ & $4.64 \cdot 10^{-3}$ & $3.33 \cdot 10^{-3}$ \\
\hline 31000 & $3.75 \cdot 10^{0}$ & $1.77 \cdot 10^{-2}$ & $1.26 \cdot 10^{-2}$ \\
\hline
\end{tabular}

Table 1: Data for random points, $k=2$

\begin{tabular}{|r|c|c|c|}
\hline$n$ & naive & tree & div-conq \\
\hline 100 & $5.00 \cdot 10^{-5}$ & $5.10 \cdot 10^{-5}$ & $4.70 \cdot 10^{-5}$ \\
\hline 310 & $5.21 \cdot 10^{-4}$ & $2.16 \cdot 10^{-4}$ & $1.96 \cdot 10^{-4}$ \\
\hline 1000 & $5.47 \cdot 10^{-3}$ & $8.59 \cdot 10^{-4}$ & $7.75 \cdot 10^{-4}$ \\
\hline 3100 & $5.15 \cdot 10^{-2}$ & $3.02 \cdot 10^{-3}$ & $2.68 \cdot 10^{-3}$ \\
\hline 10000 & $5.33 \cdot 10^{-1}$ & $1.10 \cdot 10^{-2}$ & $1.01 \cdot 10^{-2}$ \\
\hline 31000 & $5.12 \cdot 10^{0}$ & $4.49 \cdot 10^{-2}$ & $4.18 \cdot 10^{-2}$ \\
\hline
\end{tabular}

Table 2: Data for random points, $k=3$

\begin{tabular}{|r|c|c|c|}
\hline$n$ & naive & tree & div-conq \\
\hline 100 & $6.70 \cdot 10^{-5}$ & $2.40 \cdot 10^{-4}$ & $2.08 \cdot 10^{-4}$ \\
\hline 310 & $6.74 \cdot 10^{-4}$ & $9.69 \cdot 10^{-4}$ & $8.09 \cdot 10^{-4}$ \\
\hline 1000 & $6.94 \cdot 10^{-3}$ & $4.00 \cdot 10^{-3}$ & $3.36 \cdot 10^{-3}$ \\
\hline 3100 & $6.65 \cdot 10^{-2}$ & $1.56 \cdot 10^{-2}$ & $1.27 \cdot 10^{-2}$ \\
\hline 10000 & $6.85 \cdot 10^{-1}$ & $7.15 \cdot 10^{-2}$ & $5.03 \cdot 10^{-2}$ \\
\hline 31000 & $6.59 \cdot 10^{0}$ & $3.57 \cdot 10^{-1}$ & $2.05 \cdot 10^{-1}$ \\
\hline
\end{tabular}

Table 3: Data for random points, $k=4$

\begin{tabular}{|r|c|c|c|}
\hline$n$ & naive & tree & div-conq \\
\hline 100 & $8.50 \cdot 10^{-5}$ & $1.22 \cdot 10^{-3}$ & $4.71 \cdot 10^{-4}$ \\
\hline 310 & $8.05 \cdot 10^{-4}$ & $5.20 \cdot 10^{-3}$ & $2.12 \cdot 10^{-3}$ \\
\hline 1000 & $8.16 \cdot 10^{-3}$ & $2.47 \cdot 10^{-2}$ & $9.75 \cdot 10^{-3}$ \\
\hline 3100 & $7.73 \cdot 10^{-2}$ & $1.30 \cdot 10^{-1}$ & $4.09 \cdot 10^{-2}$ \\
\hline 10000 & $8.03 \cdot 10^{-1}$ & $7.11 \cdot 10^{-1}$ & $1.78 \cdot 10^{-1}$ \\
\hline 31000 & $7.71 \cdot 10^{0}$ & $3.33 \cdot 10^{0}$ & $7.53 \cdot 10^{-1}$ \\
\hline
\end{tabular}

Table 4: Data for random points, $k=5$

\begin{tabular}{|r|c|c|c|}
\hline$n$ & naive & tree & div-conq \\
\hline 100 & $9.80 \cdot 10^{-5}$ & $4.22 \cdot 10^{-3}$ & $7.77 \cdot 10^{-4}$ \\
\hline 310 & $9.22 \cdot 10^{-4}$ & $2.28 \cdot 10^{-2}$ & $3.91 \cdot 10^{-3}$ \\
\hline 1000 & $9.44 \cdot 10^{-3}$ & $1.43 \cdot 10^{-1}$ & $2.00 \cdot 10^{-2}$ \\
\hline 3100 & $9.38 \cdot 10^{-2}$ & $7.99 \cdot 10^{-1}$ & $9.35 \cdot 10^{-2}$ \\
\hline 10000 & $9.32 \cdot 10^{-1}$ & $4.60 \cdot 10^{0}$ & $4.51 \cdot 10^{-1}$ \\
\hline 31000 & $8.95 \cdot 10^{0}$ & $2.33 \cdot 10^{1}$ & $2.06 \cdot 10^{0}$ \\
\hline
\end{tabular}

Table 5: Data for random points, $k=6$ 
Finally, thanks for the reviewers who mentioned that in practical applications the fixed set typically does not change and its size is often much larger than the size of any moving set, the efficient way to preprocess the fixed set and to speed up evaluation of moving sets is one more necessary piece of the future work. This may be a good showcase for the tree-based orthant search algorithm, as in this case most of the time will be spent in answering queries and not during construction of the tree, which can be done once.

The source code as well as full logs of experiments can be found at GitHub. ${ }^{1}$

\section{Acknowledgments}

This work was financially supported by the Government of Russian Federation, Grant 074-U01.

\section{REFERENCES}

[1] A. Auger, J. Bader, D. Brockhoff, and E. Zitzler. Theory of the hypervolume indicator: Optimal $\mu$-distributions and the choice of the reference point. In Proceedings of Foundations of Genetic Algorithms, pages 87-102. ACM, 2009.

[2] A. Auger, J. Bader, D. Brockhoff, and E. Zitzler. Hypervolume-based multiobjective optimization: Theoretical foundations and practical implications. Theoretical Computer Science, 425:75-103, 2012.

[3] J. Bader and E. Zitzler. HypE: An algorithm for fast hypervolume-based many-objective optimization. Evolutionary Computation, 19(1):45-76, 2011.

[4] K. Bringmann and T. Friedrich. Approximating the least hypervolume contributor: NP-hard in general, but fast in practice. In Proceedings of the 5th International Conference on Evolutionary Multi-Criterion Optimization, pages 6-20, 2009.

[5] M. Buzdalov and A. Shalyto. A provably asymptotically fast version of the generalized Jensen algorithm for non-dominated sorting. In Parallel Problem Solving from Nature - PPSN XIII, number 8672 in Lecture Notes in Computer Science, pages 528-537. Springer, 2014.

[6] P. M. Fenwick. A new data structure for cumulative frequency tables. Software: Practice and Experience, 24(3):327-336, 1994.

\footnotetext{
${ }^{1}$ https://github.com/mbuzdalov/papers/tree/master/2016gecco-epsilon
}

[7] F.-A. Fortin, S. Grenier, and M. Parizeau. Generalizing the improved run-time complexity algorithm for non-dominated sorting. In Proceedings of Genetic and Evolutionary Computation Conference, pages 615-622. ACM, 2013.

[8] H. N. Gabow, J. L. Bentley, and R. E. Tarjan. Scaling and related techniques for geometry problems. In Proceedings of the sixteenth annual ACM symposium on Theory of computing, pages 135-143, 1984.

[9] M. T. Jensen. Reducing the run-time complexity of multiobjective EAs: The NSGA-II and other algorithms. Transactions on Evolutionary Computation, 7(5):503-515, 2003.

[10] R. Lacour, K. Klamroth, and C. M. Fonseca. A box decomposition algorithm to compute the hypervolume indicator. http://arxiv.org/abs/1510.01963, 2015.

[11] A. J. Nebro and J. J. Durillo. On the effect of applying a steady-state selection scheme in the multi-objective genetic algorithm NSGA-II. In Nature-Inspired Algorithms for Optimisation, number 193 in Studies in Computational Intelligence, pages 435-456. Springer Berlin Heidelberg, 2009.

[12] Y. Nekrich. A fast algorithm for three-dimensional layers of maxima problem. In Algorithms and Data Structures, number 6844 in Lecture Notes in Computer Science, pages 607-618. 2011.

[13] L. Russo and A. Francisco. Quick hypervolume. IEEE Transactions on Evolutionary Computation, 18(4), 2014.

[14] P. Van Emde Boas, R. Kaas, and E. Zijlstra. Design and implementation of an efficient priority queue. Mathematical Systems Theory, 10:99-127, 1976.

[15] E. Zitzler and S. Künzli. Indicator-based selection in multiobjective search. In Parallel Problem Solving from Nature - PPSN VIII, number 3242 in Lecture Notes in Computer Science, pages 832-842. 2004.

[16] E. Zitzler and L. Thiele. Multiobjective evolutionary algorithms: A comparative case study and the Strength Pareto approach. IEEE Transactions on Evolutionary Computation, 3(4):257-271, 1999.

[17] E. Zitzler, L. Thiele, M. Laumanns, C. M. Fonseca, and V. Grunert da Fonseca. Performance assessment of multiobjective optimizers: An analysis and review. IEEE Transactions on Evolutionary Computation, $7(2): 117-132,2003$. 